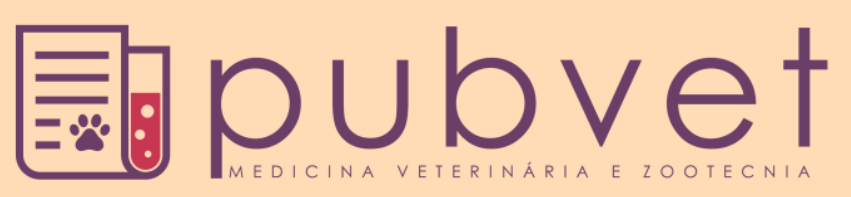

HTTP://DX.DOI.ORG/10.22256/PUBVET.V11N9.928-934

\title{
Troponina, biomarcador de injúria cardíaca, na medicina veterinária: Revisão
}

\author{
Andréia Régis de Assis ${ }^{1}$, Kelly Cristina da Silva Godoy ${ }^{1 *}$, Tamires Ramborger Antunes ${ }^{1}$, Paulo \\ Henrique Braz ${ }^{1}$, Gustavo Gomes de Oliveira ${ }^{2}$, Polyana Mayume Pereira da Silva ${ }^{3}$, Alda Izabel de \\ Souza $^{4}$
}

${ }^{I}$ Médico/a Veterinária. Doutorando/a do Programa de pós-graduação em Ciência Animal, Faculdade de Medicina Veterinária e Zootecnia (FAMEZ), Universidade Federal de Mato Grosso do Sul (UFMS). Campo Grande-MS-Brasil. E-mail: andreiaregisdeassis@gmail.com; k.c.s.godoy@gmail.com*e Tamires_ramborger_antunes@hotmail.com

${ }^{2}$ Médico veterinário. Mestrando do Programa de pós-graduação em Ciências Veterinárias, Faculdade de Medicina Veterinária e Zootecnia (FAMEZ), Universidade Federal de Mato Grosso do Sul (UFMS). Campo Grande-MS-Brasil. E-mail: gustavogomesde@hotmail.com

${ }^{3}$ Médica veterinária. Residente do Programa de Residência Profissional em Saúde-Medicina Veterinária-Universidade Federal de Mato Grosso do Sul (UFMS.Campo Grande-MS-Brasil.E-mail: polyana_mayume@hotmail.com

${ }^{4}$ Professora da Faculdade de Medicina Veterinária e Zootecnia (FAMEZ), Universidade Federal de Mato Grosso do Sul (UFMS). Campo Grande-MS-Brasil.E-mail: alda.izabel@ufms.br

RESUMO. As lesões no miocárdio são de difícil diagnóstico e podem ser confundidas com outras enfermidades, portanto é importante a associação de diferentes métodos de diagnósticos para a conclusão. Na medicina humana as troponinas são reconhecidas, há vários anos, como biomarcadores sensíveis e específicos na identificação de lesões miocárdica. As troponinas são expressas exclusivamente pelo miocárdio e liberadas para circulação logo após uma injúria no músculo cardíaco. Essa revisão aborda os diferentes tipos de troponinas, dando enfoque a sua aplicabilidade e comportamento como marcador de lesão tecidual em animais. Constatou-se se que o uso da troponina I cardíaca (cTnI), como biomarcador de injuria cardíaca, foi validada para o uso diagnóstico em diferentes espécies animais e é capaz de colaborar no diagnóstico e prognóstico das enfermidades miocárdicas, embora, não determinem o mecanismo da lesão.

Palavras chave. Animais, biomarcadores, cTnI, isoformas cardíacas, miocárdio

\section{Troponin, a cardiac injury biomarker, in veterinary medicine: Review}

ABSTRACT. Lesions in the myocardium are difficult to diagnose and can be confused with other illnesses, so it is important to combine several diagnostic methods for accuracy. In human medicine, troponins have been recognized for several years as sensitive and specific biomarkers for the identification of myocardial injury. Troponins are exclusively expressed by cardiac myocytes and released into the circulation after an injury to the heart muscle. This review different types of troponins, focusing on their applicability and behavior as a marker of cardiac injury in animals. It was found that the use of cardiac troponin I (cTnI) as a biomarker of cardiac injury has been validated for diagnostic use in several animal species and can assist in diagnosis and prognostication of myocardial diseases though, but does not specify the mechanism of injury.

Keywords: Animals, biomarkers, cTnI, cardiac isoforms, myocardium

\section{Troponina, biomarcador de injuria cardiaca, en medicina veterinaria: Revisión}

RESUMEN. Las lesiones en el miocardio son de difícil diagnóstico y pueden ser confundidas con otras enfermedades, por lo que es importante la asociación de diferentes 
métodos diagnósticos para la conclusión. En la medicina humana las troponinas son reconocidas hace varios años como biomarcadores sensibles y específicos en la identificación de lesiones miocárdicas. Las troponinas se expresan exclusivamente por el miocardio y se liberan inmediatamente después de una injuria en el músculo cardíaco. Esta revisión aborda los diferentes tipos de troponinas, dando enfoque a su aplicabilidad y comportamiento como marcador de lesión tisular en animales. Se constató que el uso de la troponina I cardiaca (cTnI), como biomarcador de injuria cardiaca, fue validado para el uso diagnóstico en diferentes especies animales y es capaz de colaborar en el diagnóstico y pronóstico de las enfermedades miocárdicas aunque, no determinen el mecanismo de la lesión.

Palabras clave: Animales, biomarcadores, cTnI, isoformas cardiacas, miocardio

\section{Introdução}

Troponinas são proteínas presentes nos sarcômeros dos músculos estriados. Fazem parte de um complexo regulador de contração e relaxamento das fibras musculares, dependentes da concentração de cálcio. Apresentam três subunidades proteicas $(\mathrm{C}, \mathrm{T}$ e I) com diferentes funções (Ebashi and Endo, 1968, Margossian and Cohen, 1973).

A determinação da concentração sérica das isoformas das troponinas de origem cardíaca (cTnT e cTnI) é considerada uma biomarcação altamente específica e sensível para lesão celular em injúrias cardíacas de mamíferos. Grande quantidade da proteína é liberada para a corrente sanguínea logo após trauma do miocárdio e permanece elevada por até duas semanas, utilizada como teste ouro na detecção de infarto agudo do miocárdio (Godoy et al., 1998, Miranda and Lima, 2014).

O uso da troponina cardíaca na medicina é bastante estudado e se tornou o teste de eleição para pacientes suspeitos de acometimento de lesão miocárdica, além de monitoramento de cardiotoxidade em pacientes em tratamento para câncer. Na medicina veterinária diversas pesquisas investigaram sua eficácia em diferentes espécies animais (DeFrancesco et al., 2002, Oyama and Sisson, 2004, Godoy et al., 2016).

Tendo em vista os recentes avanços no uso desses biomarcadores na investigação de enfermidades nos animais domésticos, esse trabalho teve por objetivo abordar os diferentes tipos de troponinas, dando enfoque a sua aplicabilidade e comportamento como marcador de lesão tecidual cardíaca.

\section{Biologia da troponina}

A Troponina é uma proteína constituída por um complexo de três polipeptídios: troponina-C
(TnC), troponina-T (TnT) e troponina-I (TnI). Cada uma dessas frações apresenta funções específicas no processo de contração e relaxamento dos músculos estriados. Estão localizadas, em aglomerados, ao redor da tropomiosina, uma fita única de proteína que envolve o filamento de actina muscular e em sua maioria, ligados a miofibrila (95\%); porém, apresentam frações livres no citosol dos miócitos (Margossian and Cohen, 1973, Filatov et al., 1999).

A TnC (troponina com afinidade pelo cálcio) possui isoformas presentes nas fibras de contração lenta do tecido muscular esquelético e cardíaco e na fibra de contração rápida do músculo esquelético. Apresenta grande afinidade com íons de cálcio. É responsável por remover os efeitos inibitórios da TnI (Filatov et al., 1999).

A TnI (Troponina com ação inibitória) apresenta três isoformas, duas de fibras esqueléticas (rápida e lenta) e uma de fibra cardíaca (cTnI) (Filatov et al., 1999) desenvolvem um obstáculo espacial que inibe a interação da actina com a miosina. Considerada uma proteína fundamental na contração muscular é capaz de inibi-la, quando a concentração de cálcio está baixa (Katrukha et al., 1998, O'Brien, 2008).

A TnT (Troponina ligada a tropomiosina) ancora o complexo da troponina à actinomiosina. $\mathrm{Na}$ formação fetal, ocorre a expressão de diversas isoformas de troponina $\mathrm{T}$ tanto em músculo cardíaco quanto em esquelético, com o desenvolvimento, permanecem três isoformas, uma para fibra de contração muscular lenta, outra para fibra de contração rápida e por fim a troponina de tecido muscular cardíaco (Cooper and Ordahl, 1985, Filatov et al., 1999).

\section{Troponinas cardíacas}

As troponinas cardíacas apresentam sequências de aminoácidos e distintos genes 
codificadores que permitem a sua identificação por testes imunológicos com anticorpos específicos (Godoy et al., 1998, Martins, 2009). A troponina I cardíaca (cTnI) possui massa molecular de $22.5 \mathrm{kDa}, 40 \%$ de diferenças entre as outras formas de troponina e uma sequência estrutural com a adição de 32 aminoácidos na cadeia $\mathrm{N}$ que determina sua especificidade cardíaca (Missov et al., 1997, Godoy et al., 1998).

Estima-se que entre 2,8\% a 4,1\% de cTnI seja citosólica, quando liberada para circulação apresenta- se na forma livre, em complexo com a troponina $\mathrm{C}$ (cTnI-C) ou em conjunto com a troponina-T e C ( cTnI-TC), assim os testes de detecção de troponina I devem identificar não somente a porção livre mas todos seus complexos (Katrukha et al., 1998). A troponina T cardiaca (cTnT) tem $37 \mathrm{kDa}$ de massa molecular e demonstra de $10-30 \%$ de diferenças estruturais entre as troponinas. Os miócitos apresentam de 6$8 \%$ de cTnT livre no citosol (Martins, 2009).

A troponina C cardíaca (cTnC), não é considerada uma troponina cardioespecífica, uma vez que apresenta estreita homologia com a troponina presente na fibra de contração lenta do tecido muscular esquelético. Não é utilizada no diagnóstico de lesão miocárdica (Filatov et al., 1999, O’Brien, 2008).

A injúria ao miocárdio leva à desestabilização e ruptura da membrana dos miócitos, com consequente extravasamento da porção citosólica das troponinas presentes no cardiomiócito. A elevação sérica ocorre entre 3 a 8 horas após a agressão, com pico em aproximadamente 18 horas. Os níveis permanecem aumentados durante uma a duas semanas. A liberação lenta da troponina ligada às miofibrilas pode justificar esse extenso tempo de permanência na circulação, já que a meia vida das troponinas e seus complexos são de apenas 2 horas (Schober et al., 1999, Miranda and Lima, 2014).

\section{Metodologia diagnóstica}

Os primeiros testes para dosagem das troponinas cTnI e cTnT utilizavam anticorpos inespecíficos, policlonais direcionados para várias regiões da cadeia estrutural, o que condicionava baixa sensibilidade e especificidade a estes ensaios diagnósticos (Martins, 2009). A substituição por anticorpos direcionados à região $\mathrm{N}$ da cadeia estrutural da troponina I, considerada mais estável, proporcionou maior sensibilidade e especificidade na detecção da cTnI (Katrukha et al., 1998). Na medicina as troponinas cardíaca são reconhecidas, pelo American College of Cardiology e a European Society of Cardiology, desde 2000, como teste de eleição para identificação precoce de injúria do miocárdio, utilizado frequentemente em quadro de emergência em pacientes com sinais clínicos de infarto agudo do miocárdio (Alpert et al., 2000, Miranda and Lima, 2014).

Como as proteínas da cTnI são filogeneticamente preservadas e apresentam uma homologia de $95 \%$ entre os mamíferos, sua aplicabilidade tem sido demonstrada em estudos com várias espécies animais (O’Brien, 2008, Serra et al., 2010). Para realização da investigação da concentração das troponinas séricas, são utilizados imunoensaios enzimáticos (Enzyme-Linked Immunosorbent Assay - ELISA em placa) com anticorpos específicos além de kit de teste rápido cromatográfico, comumente utilizado em quadros de emergência, podem ser quantitativo ou qualitativo, as amostras de sangue em anticoagulantes etilenodiaminotetracético (EDTA) ou heparina, soro ou plasma podem ser empregados (O’Brien, 2008, Serra et al., 2010).

A troponina $\mathrm{T}$ e I no soro se mantém estável por até $24 \mathrm{~h}$ em temperatura ambiente. Quando armazenada a $4^{\circ} \mathrm{C}$ a estabilidade se mantém por até uma semana (Archer, 2003). A conservação a $-20^{\circ} \mathrm{C}$ demonstra perdas de, aproximadamente, $10 \%$ de atividade por semana. A estabilidade da amostra é alta quando mantida a temperaturas inferiores a $-70^{\circ} \mathrm{C}$; porém ocorrem perdas significativas após vários congelamentos e descongelamentos. Presença de coágulos, fibrina, lipemia, hemólise ou anticorpos heterofílicos nas amostras promovem resultados falso positivo e podem ser amenizado pelo uso de tubos com ativador de coágulos, aumento no tempo de centrifugação diminuindo a fibrina, os anticorpos heterofilicos diminuem com o avanço das técnicas imunológicas que substituem os anticorpos monoclonais desenvolvidos em camundongos (Archer, 2003, O'Brien, 2008).

Pacientes com insuficiência renal podem apresentar aumento na concentração sérica das troponinas sem evidencias clinicas de lesão no miocárdio devido ao prejuízo da excreção renal, principal via de eliminação desses biomarcador (Porciello et al., 2008, Martins, 2009). Sleeper et al. (2001) estabeleceram valores de cTnI normais para cães entre 0,03 e $0,07 \mathrm{ng} / \mathrm{mL}$ e em gatos entre 0,03 a $0,16 \mathrm{ng} / \mathrm{mL}$. Nos equinos, em pastejo e em 
treinamento, clinicamente saudáveis, o intervalo normal variou de 0,04 a $0,11 \mathrm{ng} / \mathrm{mL}$ e em bovinos de 0,005 a $0,09 \mathrm{ng} / \mathrm{mL}$ (Fartashvand et al., 2013).

\section{Uso da troponina como marcador de doenças cardíacas primárias}

O diagnóstico da doença cardíaca é complexo, uma vez que os sinais clínicos são inespecíficos, podendo ser confundidos com outras enfermidades. Estudos realizados com dosagem sérica de cTnT e cTnI, em diferentes espécies de animais indicaram aumento devido a lesões miocárdicas. Doenças cardíacas primárias ou secundarias e o uso de fármacos cardiotóxicos foram reconhecidos como causas de aumento das troponinas (Archer, 2003, Oyama and Solter, 2004, Wallace et al., 2004, Undhad et al., 2012); contudo Alves et al. (2015) não constataram alterações no teste cromatográfico qualitativo de Troponina I em cães com cardiopatia crônica.

Estudo que comparou a eficácia da troponina e do eletrocardiograma (ECG) para diagnóstico de contusão cardíaca em cães que sofreram trauma torácico, identificou maior sensibilidade do biomarcador. $\mathrm{O}$ aumento das troponinas cardíacas (cTnI e cTnT) foi detectado em 58\% dos animais e apenas 30\% de alterações no ECG (Schober et al., 1999). O aumento sérico de cTnT e cTnI também foi associado a quadros de insuficiência cardíaca congestiva, secundária a doença valvular degenerativa, cardiomiopatia dilatada e menor tempo de sobrevivência em cães (DeFrancesco et al., 2002, Oyama and Sisson, 2004). A avaliação da cTnI demonstrou sensibilidade de $85 \%$ e especificidade de $98 \%$ na diferenciação entre felinos saudáveis e portadores de cardiomiopatia hipertrofica (Hemdon et al., 2002). Em equinos, aumento significativo de cTnI foi identificado em cardiomiopatias primárias. Elevações nas concentrações séricas foram menores em quadros de arritmias causadas por lesões miocárdicas secundárias (Nath et al., 2012). Lesões isquêmicas crônicas induzidas no miocárdio de ovinos igualmente resultaram elevações de cTnI, Em bovinos, Mellanby et al. (2009) verificaram o aumento sérico da cTnI em casos de pericardite e embora tenha indicado lesão miocárdica não permitiu a confirmação da etiologia.

\section{Aumento de troponina cardíaca em lesões miocárdicas secundárias}

Conolli et al. (2009) verificaram o aumento na cTnI em gatos com dificuldades respiratórias de origem cardíaca e não cardíaca. Nessa espécie o teste apresentou sensibilidade de 65,2\% e especificidade $90,0 \%$ na confirmação da causa cardíaca.

Diferentemente dos felinos, em cães foi possível confirmar a origem cardíaca da desordem respiratória com o uso da cTnI (Payne et al., 2011). No entanto, em ambas as espécies os autores recomendaram a associação com outros métodos diagnósticos para a confirmação da etiologia primária. Ressalta-se que, em humanos são realizadas dosagens seriadas, se após um intervalo de 10 horas seu o nível sérico diminui associa se a dispneia de origem não cardíaca, direcionando assim o tratamento inicial de forma adequada evitando complicações pelo uso incorreto da terapêutica (Martins, 2009, Payne et al., 2011).

Cães com Erlichia sp, que desenvolveram quadros anêmicos graves e síndrome da resposta inflamatória sistêmica, provocada pelo agente, apresentaram aumento na concentração sérica de cTnI (Diniz et al., 2008, Koutinas et al., 2012). Lesões cardíacas desencadeadas pela leishmaniose visceral canina também provocaram elevações da cTnI (Silvestrini et al., 2012, Godoy et al., 2016). Uma correlação entre altas concentrações séricas de troponina e mortalidade foi observado em cadelas apresentando quadro de piometra (Hagman et al., 2007).

Isquemia e necrose miocárdica por efusão pericárdica idiopática ou hemangiossarcoma, em cães, apresentaram aumento nas concentrações da cTnT e cTnI, com diferença estatistica entre elas. Os valores da cTnI foram significantemente mais altos quando a efusão foi causada pelo hemangiossarcoma (Shaw et al., 2004). Posteriormente, Linde et al. (2006) constataram o aumento da cTnI no plasma e na efusão pericárdica de cães, porém sem diferenciar causa neoplásica ou não neoplásica. Gatos e cães com insuficiência renal azotêmica e cães com outras doenças sistêmicas não cardíacas, elevaram a concentração de cTnI, sugerindo lesão ao miocárdio não identificadas clinicamente (Porciello et al., 2008).

Verbiest et al. (2013) submeteram cães a cirurgia ortopédicas e estes apresentaram uma concentração de cTnI pós-anestésica maior do que a concentração pré-anestésica de cTnI diferentemente de equinos sadios, submetidos a anestesia geral que não aumentaram a concentração de cTnI até 24 h do pós-operatório 
(Slack et al., 2011). O tratamento com doxorrubicina elevou as concentrações séricas cTnT em cães sem mudanças clinicas detectáveis, o eletrocardiograma e o ecocardiograma não foram sugestivos de cardiotoxicidade (DeFrancesco et al., 2002).

Variações nas concentrações das troponinas foram identificadas em casos de ofidismo em equinos. Em animais endotoxêmicos, a cTnI foi considerada útil na detecção de dano cardíaco antes de alterações ventriculares detectáveis no eletrocardiograma (Nostell et al., 2012). Segundo Radcliffe et al. (2012) elevações na cTnI foi indicador de mau prognóstico, em cavalos doentes, após a cirurgia abdominal de emergência.

Quadro de anemia e intenso parasitismo associados a teileriose em bovinos promoveu aumento da concentração sérica da cTnI. A proporção desse aumento foi correlacionada à gravidade da doença nesses animais (Fartashvand et al., 2013). Bezerros com febre aftosa tiveram aumento na concentração sérica da cTnI atribuída a lesão do miocárdio que foi confirmada pela redução da imunomarcação para troponina em cardiomiócitos (Tunca et al., 2008). Em ovinos, elevações das concentrações de cTnT e cTnI foram associadas à degeneração hialina e necrose de tecido muscular esquelético, identificada pelo exame histopatológico como doença do músculo branco (Gunes et al., 2010)

\section{Considerações finais}

O uso da troponina como cardiomarcador na medicina veterinária ainda é pouco difundido e apesar desse biomarcador não determinar o mecanismo da lesão, quando realizado de maneira seriada, pode tornar-se uma importante ferramenta diagnóstica no monitoramento do tratamento e prognóstico de pacientes com cardiopatias nas diferentes espécies.

Agradecimentos: À Fundação de Apoio ao Ensino, Ciência e Tecnologia de Mato Grosso do Sul (FUNDECT), pelo apoio financeiro.

\section{Referências Bibliográficas}

Alpert, J. S., Thygesen, K., Antman, E. \& Bassand, J. P. 2000. Myocardial infarction redefined. European Heart Journal, 21, 15021513.

Alves, A. C., Sousa, V. R. F., Silva, E. P., Néspoli, P. E. B., Silva, F. G. \& Almeida, A. B. P. F. 2015. Uso de biomarcadores CK-NAC, CK-
MB e tropina I em cães com doença cardáica. Archives of Veterinary Science, 20, 103-108.

Archer, J. 2003. Cardiac biomarkers: a review. Comparative Clinical Pathology, 12, 121-128.

Cooper, T. A. \& Ordahl, C. P. 1985. A single cardiac troponin $\mathrm{T}$ gene generates embryonic and adult isoforms via developmentally regulated alternate splicing. Journal of Biological Chemistry, 260, 11140-11148.

DeFrancesco, T. C., Atkins, C. E., Keene, B. W., Coats, J. R. \& Hauck, M. L. 2002. Prospective clinical evaluation of serum cardiac troponin $\mathrm{T}$ in dogs admitted to a veterinary teaching hospital. Journal of Veterinary Internal Medicine, 16, 553-557.

Diniz, P. P. V. P., De Morais, H. S. A., Breitschwerdt, E. B. \& Schwartz, D. S. 2008. Serum cardiac troponin I concentration in dogs with ehrlichiosis. Journal of Veterinary Internal Medicine, 22, 1136-1143.

Ebashi, S. \& Endo, M. A. 1968. Calcium and muscle contraction. Progress in Biophysics and Molecular Biology, 18, 123-166.

Fartashvand, M., Nadalian, M. G., Sakha, M. \& Safi, S. 2013. Elevated serum cardiac troponin I in cattle with theileriosis. Journal of Veterinary Internal Medicine, 27, 194-199.

Filatov, V. L., Katrukha, A. G., Bulargina, T. V. \& Gusev, N. B. 1999. Troponin: structure, properties, and mechanism of functioning. Biochemistry, 64, 969-985.

Godoy, K. C. S., Braz, P. H., Assis, A. R., Antunes, T. R., Gomes, D. C. \& Souza, A. I. 2016. Avaliação dos indicadores de lesão miocárdica em cães com leishmaniose visceral. Arquivo Brasileiro de Medicina Veterinaria e Zootecnia, 68, 313-320.

Godoy, M. F., Braile, D. M. \& Purini Neto, J. 1998. A troponina como marcador de injúria celular miocárdica. Arquivos Brasileiros de Cardiologia, 71, 629-633.

Gunes, V., Ozcan, K., Citil, M., Onmaz, A. C. \& Erdogan, H. M. 2010. Detection of myocardial degeneration with point-of-care cardiac troponin assays and histopathology in lambs with white muscle disease. The Veterinary Journal, 184, 376-378.

Hagman, R., Lagerstedt, A.-S., Fransson, B. A., Bergström, A. \& Häggström, J. 2007. Cardiac troponin I levels in canine pyometra. Acta Veterinaria Scandinavica, 49, 1-8. 
Hemdon, W. E., Kittleson, M. D., Sanderson, K., Drobatz, K. J., Clifford, C. A., Gelzer, A., Summerfield, N. J., Linde, A. \& Sleeper, M. M. 2002. Cardiac troponin I in feline hypertrophic cardiomyopathy. Journal of Veterinary Internal Medicine, 16, 558-564.

Katrukha, A. G., Bereznikova, A. V., Filatov, V. L., Esakova, T. V., Kolosova, O. V., Pettersson, K., Lövgren, T., Bulargina, T. V., Trifonov, I. R. \& Gratsiansky, N. A. 1998. Degradation of cardiac troponin I: implication for reliable immunodetection. Clinical Chemistry, 44, 2433-2440.

Koutinas, C. K., Mylonakis, M. E., O’Brien, P. J., Leontides, L., Siarkou, V. I., Breitschwerdt, E. B. \& Koutinas, A. F. 2012. Serum cardiac troponin I concentrations in naturally occurring myelosuppressive and non-myelosuppressive canine monocytic ehrlichiosis. The Veterinary Journal, 194, 259-261.

Linde, A., Summerfield, N. J., Sleeper, M. M., Wright, F. B., Clifford, C. A., Melgarejo, T. \& Knight, D. H. 2006. Pilot study on cardiac troponin I levels in dogs with pericardial effusion. Journal of Veterinary Cardiology, 8, 19-23.

Margossian, S. S. \& Cohen, C. 1973. Troponin subunit interactions. Journal of Molecular Biology, 81, 409-413.

Martins, C. S. 2009. Troponina: estrutura, fisiopatologia e importância clínica para além da isquemia miocárdica. Arquivos de Medicina, 23, 221-240.

Mellanby, R. J., Henry, J. P., Cash, R., Ricketts, S. W., Bexiga, R., Truyers, I. \& Mellor, D. J. 2009. Serum cardiac troponin I concentrations in cattle with cardiac and noncardiac disorders. Journal of Veterinary Internal Medicine, 23, 926-930.

Miranda, M. R. \& Lima, L. M. 2014. Marcadores bioquímicos do infarto agudo do miocárdio. Revista Médica de Minas Gerais, 24, 98-105.

Missov, E., Calzolari, C. \& Pau, B. 1997. Circulating cardiac troponin $\mathrm{I}$ in severe congestive heart failure. Circulation, 96, 29532958.

Nath, L. C., Anderson, G. A., Hinchcliff, K. W. \& Savage, C. J. 2012. Serum cardiac troponin I concentrations in horses with cardiac disease. Australian Veterinary Journal, 90, 351-357.

Nostell, K., Bröjer, J., Höglund, K., Edner, A. \& Häggström, J. 2012. Cardiac troponin I and the occurrence of cardiac arrhythmias in horses with experimentally induced endotoxaemia. The Veterinary Journal, 192, 171-175.

O'Brien, P. J. 2008. Cardiac troponin is the most effective translational safety biomarker for myocardial injury in cardiotoxicity. Toxicology, 245, 206-218.

Oyama, M. A. \& Sisson, D. D. 2004. Cardiac troponin-I concentration in dogs with cardiac disease. Journal of Veterinary Internal Medicine, 18, 831-839.

Oyama, M. A. \& Solter, P. F. 2004. Validation of an immunoassay for measurement of canine cardiac troponin-I. Journal of Veterinary Cardiology, 6, 17-24.

Payne, E. E., Roberts, B. K., Schroeder, N., Burk, R. L. \& Schermerhorn, T. 2011. Assessment of a point-of-care cardiac troponin I test to differentiate cardiac from noncardiac causes of respiratory distress in dogs. Journal of Veterinary Emergency and Critical Care, 21, 217-225.

Porciello, F., Rishniw, M., Herndon, W. E., Birettoni, F., Antognoni, M. T. \& Simpson, K. W. 2008. Cardiac troponin I is elevated in dogs and cats with azotaemia renal failure and in dogs with non-cardiac systemic disease. Australian Veterinary Journal, 86, 390-394.

Radcliffe, R. M., Divers, T. J., Fletcher, D. J., Mohammed, H. \& Kraus, M. S. 2012. Evaluation of L-lactate and cardiac troponin I in horses undergoing emergency abdominal surgery. Journal of Veterinary Emergency and Critical Care, 22, 313-319.

Schober, K. E., Kirbach, B. \& Oechtering, G. 1999. Noninvasive assessment of myocardial cell injury in dogs with suspected cardiac contusion. Journal of Veterinary Cardiology, 1, 17-25.

Serra, M., Papakonstantinou, S., Adamcova, M. \& O'Brien, P. J. 2010. Veterinary and toxicological applications for the detection of cardiac injury using cardiac troponin. The Veterinary Journal, 185, 50-57.

Shaw, S. P., Rozanski, E. A. \& Rush, J. E. 2004. Cardiac troponins $\mathrm{I}$ and $\mathrm{T}$ in dogs with pericardial effusion. Journal of Veterinary Internal Medicine, 18, 322-324.

Silvestrini, P., Piviani, M., Alberola, J., Rodríguez-Cortés, A., Planellas, M., Roura, X., O'brien, P. J. \& Pastor, J. 2012. Serum cardiac troponin I concentrations in dogs with 
leishmaniasis: correlation with age and clinicopathologic abnormalities. Veterinary Clinical Pathology, 41, 568-574.

Slack, J., Boston, R., Driessen, B. \& Reef, V. 2011. Effect of general anesthesia on plasma cardiac troponin I concentrations in healthy horses. Journal of Veterinary Cardiology, 13, 163-169.

Sleeper, M. M., Clifford, C. A. \& Laster, L. L. 2001. Cardiac troponin I in the normal dog and cat. Journal of Veterinary Internal Medicine, 15, 501-503.

Tunca, R., Sozmen, M., Erdogan, H., Citil, M., Uzlu, E., Ozen, H. \& Gokçe, E. 2008. Determination of cardiac troponin I in the blood and heart of calves with foot-and-mouth disease. Journal of Veterinary Diagnostic Investigation, 20, 598-605.

Undhad, V. V., Fefar, D. T., Jivani, B. M., Gupta, H., Ghodasara, D. J., Joshi, B. P. \& Prajapati, K. S. 2012. Cardiac troponin: an emerging cardiac biomarker in animal health. Veterinary World, 5, 508-511.

Verbiest, T., Binst, D., Waelbers, T., Coppieters, E. \& Polis, I. 2013. Perioperative changes in cardiac troponin I concentrations in dogs. Research in Veterinary Science, 94, 446-448.

Wallace, K. B., Hausner, E., Herman, E., Holt, G. D., Macgregor, J. T., Metz, A. L., Murphy, E., Rosenblum, I., Y,, Sistare, F. D. \& York, M. J. 2004. Serum troponins as biomarkers of druginduced cardiac toxicity. Toxicologic Pathology, 32, 106-121.

\section{Article History:}

Received 17 May 2017

Accepted 07 June 2017

Available online 8 Augusto 2017

License information: This is an open-access article distributed under the terms of the Creative Commons Attribution License 4.0, which permits unrestricted use, distribution, and reproduction in any medium, provided the original work is properly cited 